Acta Crystallographica Section E

Structure Reports

Online

ISSN 1600-5368

\section{Michael Schaefer, ${ }^{a}$ Daniel Kurowski, ${ }^{\text {b }}$ Arno Pfitzner, Christian Näther ${ }^{\mathrm{a}}$ and Wolfgang Bensch $^{\mathrm{a}}$}

${ }^{a}$ Institut für Anorganische Chemie, ChristianAlbrechts-Universität Kiel, Olshausenstraße 40, D-24098 Kiel, Germany, and ${ }^{\mathbf{b}}$ Institut für Anorganische Chemie, Universität Regensburg, Universitätsstraße 31, D-93040 Regensburg, Germany

\section{Key indicators}

Single-crystal X-ray study

$T=293 \mathrm{~K}$

Mean $\sigma(\mathrm{C}-\mathrm{C})=0.008 \AA$

$\mathrm{H}$-atom completeness $97 \%$

Disorder in solvent or counterion

$R$ factor $=0.028$

$w R$ factor $=0.083$

Data-to-parameter ratio $=26.6$

For details of how these key indicators were automatically derived from the article, see http://journals.iucr.org/e.

\title{
[Tris(2-aminoethyl)amine]manganese(II) heptasulfidotetraantimony(III) hemihydrate
}

Yellow crystals of the title compound, $\left[\mathrm{Mn}(\operatorname{dien})_{2}\right]\left[\mathrm{Sb}_{4} \mathrm{~S}_{7}\right] \cdot$ $0.5 \mathrm{H}_{2} \mathrm{O}$ (dien = diethylenetriamine $=\mathrm{C}_{4} \mathrm{H}_{13} \mathrm{~N}_{3}$ ) were synthesized under solvothermal conditions by reacting $\mathrm{MnSb}_{2} \mathrm{~S}_{4}$ with pure dien. The $\mathrm{Mn}^{2+}$ ion is in an octahedral environment of six $\mathrm{N}$ atoms of the tridentate dien molecules. The $\left[\mathrm{Sb}_{4} \mathrm{~S}_{7}\right]^{2-}$ units, and anions are joined together to form two-dimensional layers. In the layered anion, $\mathrm{Sb}_{2} \mathrm{~S}_{2}, \mathrm{Sb}_{4} \mathrm{~S}_{4}$ and $\mathrm{Sb}_{8} \mathrm{~S}_{8}$ heterorings are found.

\section{Comment}

Until now, more than a dozen thioantimonates(III) with the $\left[\mathrm{Sb}_{4} \mathrm{~S}_{7}\right]^{2-}$ anion with different dimensionalities have been reported (Sheldrick \& Häusler, 1988; Dittmar \& Schäfer, 1978a,b; Cordier et al., 1984; Dittmar \& Schäfer, 1977; Bensch \& Schur, 1997; Stephan \& Kanatzidis, 1997). For example, in $\mathrm{K}_{2} \mathrm{Sb}_{4} \mathrm{~S}_{7}$ (Graf \& Schäfer, 1972), a three-dimensional network is observed. Incorporation of water yields a layered $\left[\mathrm{Sb}_{4} \mathrm{~S}_{7}\right]^{2-}$ anion (Eisenmann \& Schäfer, 1979). To the best of our knowledge, most of the $\left[\mathrm{Sb}_{4} \mathrm{~S}_{7}\right]^{2-}$ compounds were prepared by reacting elements under solvothermal conditions or in a reactive flux. During our systematic search for new materials which are suitable as educts for solvothermal reactions, we synthesized the layered compound [Mn(dien $\left.)_{2}\right]-$ $\mathrm{Sb}_{4} \mathrm{~S}_{7} \cdot 0.5 \mathrm{H}_{2} \mathrm{O}$, (I), applying the ternary compound $\mathrm{MnSb}_{2} \mathrm{~S}_{4}$ and dien as the solvent.

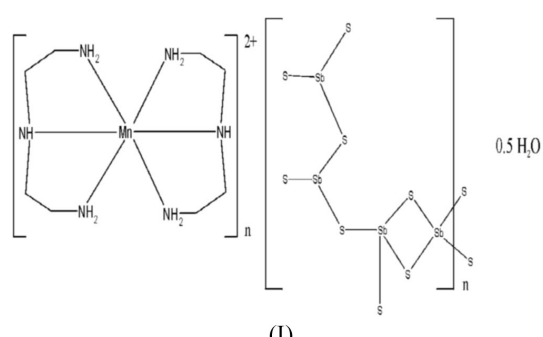

(I)

The structure of (I) is composed of isolated $\left[\mathrm{Mn}(\mathrm{dien})_{2}\right]^{2+}$ cations and layered $\left[\mathrm{Sb}_{4} \mathrm{~S}_{7}\right]^{2-}$ anions (Fig. 1). The $\mathrm{Mn}^{2+}$ ion is surrounded by six $\mathrm{N}$ atoms of two dien ligands forming a distorted octahedron $\left(\mathrm{MnN}_{6}\right)$, which adopts the mer configuration. The $\mathrm{Mn}-\mathrm{N}$ distances range from 2.241 (4) to 2.326 (4) $\AA$, with $\mathrm{N}-\mathrm{Mn}-\mathrm{N}$ angles between 75.2 (1) and $176.2(1)^{\circ}$. All values are in the normal ranges for $\mathrm{MnN}_{6}$ octahedra (von Zelewsky, 1996; Ellermeier \& Bensch, 2002; Schaefer et al., 2003). In the anionic part of the structure, two $\mathrm{SbS}_{3}$ trigonal pyramids and two $\mathrm{SbS}_{4}$ units are the primary building units. Both $\mathrm{SbS}_{3}$ moieties share a common corner, forming an $\mathrm{Sb}_{2} \mathrm{~S}_{5}$ unit. The $\mathrm{Sb}-\mathrm{S}$ bond lengths vary between 2.388 (1) and 2.479 (1) $\AA$, with $\mathrm{S}-\mathrm{Sb}-\mathrm{S}$ angles between 90.01 (3) and 104.79 (4) $)^{\circ}$. The two $\mathrm{SbS}_{4}$ units share a common
Received 17 November 2003 Accepted 4 December 2003 Online 17 January 2004 


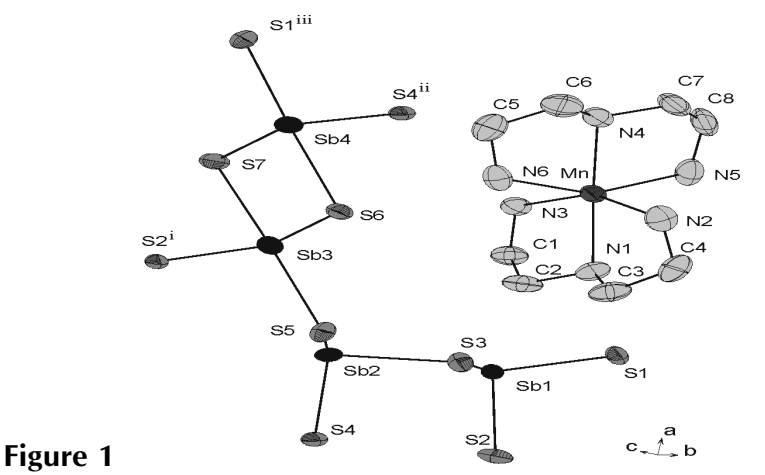

The asymmetric unit of $\left[\mathrm{Mn}(\operatorname{dien})_{2}\right] \mathrm{Sb}_{4} \mathrm{~S}_{7} \cdot 0.5 \mathrm{H}_{2} \mathrm{O}$, together with some symmetry-equivalent $\mathrm{S}$ atoms to complete the coordination of $\mathrm{Sb}$. Displacement ellipsoids are drawn at the $50 \%$ probability level. The symmetry codes are given in Table $1 . \mathrm{H}$ atoms have been omitted.

edge, forming an $\mathrm{Sb}_{2} \mathrm{~S}_{2}$ hetero-ring within a $\mathrm{Sb}_{2} \mathrm{~S}_{6}$ unit. In both $\mathrm{SbS}_{4}$ units, two longer distances are found [2.692(1) and 2.832 (1) $\AA$ for Sb3, and 2.728 (1) and 2.746 (1) $\AA$ for Sb4]. The longer separations are in trans-position to each other, with angles of $171.92(3)^{\circ}$ for $\mathrm{S} 5-\mathrm{Sb} 3-\mathrm{S} 7$ and $174.53(3)^{\circ}$ for $\mathrm{S} 6-$ $\mathrm{Sb} 4-\mathrm{S} 1^{\mathrm{iii}}$ (see Table 1). Angles and distances are comparable with those reported in the literature (Sheldrick \& Häusler, 1988; Dittmar \& Schäfer, 1978a,b; Cordier et al., 1984; Schur \& Bensch, 1997). The secondary building blocks $\mathrm{Sb}_{2} \mathrm{~S}_{5}$ and $\mathrm{Sb}_{2} \mathrm{~S}_{6}$ share a common corner (S5) and further condensation leads to $\mathrm{Sb}_{4} \mathrm{~S}_{4}$ and $\mathrm{Sb}_{8} \mathrm{~S}_{8}$ rings. The eight-membered rings are fused to each other parallel to the $a$ and $b$ axis (Fig. 2). The mer$\left[\mathrm{Mn}(\operatorname{dien})_{2}\right]^{2+}$ cations and the water molecules are found above and below the $\mathrm{Sb}_{8} \mathrm{~S}_{8}$ hetero-rings. The coordination numbers of the $\mathrm{Sb} 1$ and $\mathrm{Sb} 2$ atoms are enhanced from three to four by $\mathrm{S}$ atoms at longer distances of $3.118(1)$ and 3.264 (1) $\AA$, respectively. We note that (I) is isostructural with $\left[\mathrm{Ni}(\text { dien })_{2}\right] \mathrm{Sb}_{4} \mathrm{~S}_{7} \cdot \mathrm{H}_{2} \mathrm{O}$ (Stähler et al., 2003). The unit-cell volume of the latter compound is $40 \AA^{3}$ smaller than that of the title compound.

\section{Experimental}

[Mn(dien $\left.)_{2}\right] \mathrm{Sb}_{4} \mathrm{~S}_{7} \cdot 0.5 \mathrm{H}_{2} \mathrm{O}$ was obtained in nearly $30 \%$ yield by the reaction of $\mathrm{MnSb}_{2} \mathrm{~S}_{4}$ (Pfitzner \& Kurowski, 2000) (0.100 g, $0.234 \mathrm{mmol}$ ) in a pure solution of $3 \mathrm{ml}$ dien (99\%) (Merck). The mixture was heated in Teflon-lined steel autoclaves with an inner volume of $30 \mathrm{ml}$ for $14 \mathrm{~d}$ at $403 \mathrm{~K}$ and then cooled to room temperature within $3 \mathrm{~h}$. After washing with water, dried under vacuum, yellow crystals of $\left[\mathrm{Mn}(\text { dien })_{2}\right] \mathrm{Sb}_{4} \mathrm{~S}_{7} \cdot 0.5 \mathrm{H}_{2} 0$ were obtained. The compound is stable in air and water. Analysis calculated for $\left[\mathrm{Mn}(\text { dien })_{2}\right] \mathrm{Sb}_{4} \mathrm{~S}_{7} .0 .5 \mathrm{H}_{2} 0$ : C 9.78, $\mathrm{H} 2.87, \mathrm{~N} 8.55, \mathrm{~S} 22.84 \%$; found: $\mathrm{C}$ 9.59, H 2.61, N 8.75, S 22.15\%.

\section{Crystal data}

$\left[\mathrm{Mn}\left(\mathrm{C}_{4} \mathrm{H}_{13} \mathrm{~N}_{3}\right)_{2}\right]\left[\mathrm{Sb}_{4} \mathrm{~S}_{7}\right] \cdot 0.5 \mathrm{H}_{2}$
$M_{r}=980.71$
Monoclinic, $P 2_{1} / c$
$a=9.7216(19) \AA$
$b=16.113(3) \AA$
$c=17.339(4) \AA$
$\beta=91.88(3)^{\circ}$
$V=2714.6(9) \AA^{3}$
$Z=4$

Data collection

$\begin{array}{ll}\text { Stoe IPDS diffractometer } & 6508 \text { independent reflections } \\ \varphi \text { scans } & 5824 \text { reflections with } I>2 \sigma(I) \\ \text { Absorption correction: numerical } & R_{\text {int }}=0.032 \\ \quad[X \text {-SHAPE (Stoe \& Cie, 1998) } & \theta_{\max }=28.0^{\circ} \\ \quad \text { and } X \text { - } R E D \text { (Stoe \& Cie, } 1998)] & h=-12 \rightarrow 12 \\ \quad T_{\min }=0.390, T_{\max }=0.609 & k=-21 \rightarrow 21 \\ 31130 \text { measured reflections } & l=-22 \rightarrow 22 \\ & \\ \text { Refinement } & w=1 /\left[\sigma^{2}\left(F_{o}{ }^{2}\right)+(0.0547 P)^{2}\right. \\ \text { Refinement on } F^{2} & \quad+3.6221 P] \\ R\left[F^{2}>2 \sigma\left(F^{2}\right)\right]=0.028 & \text { where } P=\left(F_{o}{ }^{2}+2 F_{c}{ }^{2}\right) / 3 \\ w R\left(F^{2}\right)=0.083 & (\Delta / \sigma)_{\max }=0.001 \\ S=1.05 & \Delta \rho_{\max }=1.25 \mathrm{e} \AA^{-3} \\ 6508 \text { reflections } & \Delta \rho_{\min }=-1.12 \AA^{-3} \\ 245 \text { parameters } & \text { Extinction correction: SHELXL97 } \\ \text { H-atom parameters constrained } & \text { Extinction coefficient: } 0.00169(13)\end{array}$

Table 1

Selected geometric parameters $\left(\AA,^{\circ}\right)$.

\begin{tabular}{|c|c|c|c|}
\hline $\mathrm{Mn}-\mathrm{N} 4$ & $2.241(4)$ & Sb3-S6 & $2.3893(12)$ \\
\hline $\mathrm{Mn}-\mathrm{N} 2$ & $2.256(4)$ & $\mathrm{Sb} 3-\mathrm{S} 2^{\mathrm{i}}$ & 2.4451 (11) \\
\hline $\mathrm{Mn}-\mathrm{N} 5$ & $2.259(4)$ & Sb3-S7 & 2.6917 (13) \\
\hline $\mathrm{Mn}-\mathrm{N} 1$ & $2.270(4)$ & $\mathrm{Sb} 3-\mathrm{S} 5$ & $2.8322(13)$ \\
\hline $\mathrm{Mn}-\mathrm{N} 3$ & $2.320(4)$ & $\mathrm{Sb} 4-\mathrm{S} 7$ & 2.3907 (13) \\
\hline $\mathrm{Mn}-\mathrm{N} 6$ & $2.326(4)$ & $\mathrm{Sb} 4-\mathrm{S} 4^{\mathrm{ii}}$ & $2.4495(11)$ \\
\hline $\mathrm{Sb} 1-\mathrm{S} 1$ & $2.4076(10)$ & $\mathrm{Sb} 4-\mathrm{Si}^{\mathrm{iii}}$ & 2.7279 (13) \\
\hline $\mathrm{Sb} 1-\mathrm{S} 2$ & 2.4539 (11) & Sb4-S6 & 2.7459 (13) \\
\hline $\mathrm{Sb} 1-\mathrm{S} 3$ & $2.4790(10)$ & $\mathrm{S} 1-\mathrm{Sb} 4^{\mathrm{iv}}$ & 2.7279 (13) \\
\hline $\mathrm{Sb} 2-\mathrm{S} 5$ & $2.3883(10)$ & $\mathrm{S} 2-\mathrm{Sb} 3^{\mathrm{v}}$ & 2.4451 (11) \\
\hline $\mathrm{Sb} 2-\mathrm{S} 4$ & $2.4682(11)$ & $\mathrm{S} 4-\mathrm{Sb} 4^{\mathrm{vi}}$ & $2.4495(11)$ \\
\hline $\mathrm{Sb} 2-\mathrm{S} 3$ & $2.4701(10)$ & & \\
\hline $\mathrm{N} 4-\mathrm{Mn}-\mathrm{N} 2$ & $107.09(13)$ & $\mathrm{S} 1-\mathrm{Sb} 1-\mathrm{S} 2$ & $99.30(4)$ \\
\hline $\mathrm{N} 4-\mathrm{Mn}-\mathrm{N} 5$ & 77.15 (16) & $\mathrm{S} 1-\mathrm{Sb} 1-\mathrm{S} 3$ & $92.19(4)$ \\
\hline $\mathrm{N} 2-\mathrm{Mn}-\mathrm{N} 5$ & $98.22(16)$ & $\mathrm{S} 2-\mathrm{Sb} 1-\mathrm{S} 3$ & $92.35(4)$ \\
\hline $\mathrm{N} 4-\mathrm{Mn}-\mathrm{N} 1$ & $176.26(13)$ & $\mathrm{S} 5-\mathrm{Sb} 2-\mathrm{S} 4$ & $104.82(4)$ \\
\hline $\mathrm{N} 2-\mathrm{Mn}-\mathrm{N} 1$ & $76.64(13)$ & $\mathrm{S} 5-\mathrm{Sb} 2-\mathrm{S} 3$ & $92.03(4)$ \\
\hline $\mathrm{N} 5-\mathrm{Mn}-\mathrm{N} 1$ & $102.89(16)$ & $\mathrm{S} 4-\mathrm{Sb} 2-\mathrm{S} 3$ & $91.09(4)$ \\
\hline $\mathrm{N} 4-\mathrm{Mn}-\mathrm{N} 3$ & $100.00(15)$ & $\mathrm{S} 6-\mathrm{Sb} 3-\mathrm{S} 7$ & $87.18(4)$ \\
\hline $\mathrm{N} 2-\mathrm{Mn}-\mathrm{N} 3$ & $152.13(15)$ & $\mathrm{S} 6-\mathrm{Sb} 3-\mathrm{S} 5$ & $87.83(4)$ \\
\hline $\mathrm{N} 5-\mathrm{Mn}-\mathrm{N} 3$ & $93.99(16)$ & $\mathrm{S} 7-\mathrm{Sb} 3-\mathrm{S} 5$ & $171.94(3)$ \\
\hline $\mathrm{N} 1-\mathrm{Mn}-\mathrm{N} 3$ & $76.26(15)$ & $\mathrm{S} 7-\mathrm{Sb} 4-\mathrm{S} 6$ & $85.92(4)$ \\
\hline $\mathrm{N} 4-\mathrm{Mn}-\mathrm{N} 6$ & $75.19(15)$ & $\mathrm{Sb} 2-\mathrm{S} 3-\mathrm{Sb} 1$ & $102.56(4)$ \\
\hline $\mathrm{N} 2-\mathrm{Mn}-\mathrm{N} 6$ & $90.53(14)$ & $\mathrm{Sb} 2-\mathrm{S} 5-\mathrm{Sb} 3$ & $88.68(3)$ \\
\hline $\mathrm{N} 5-\mathrm{Mn}-\mathrm{N} 6$ & $152.33(17)$ & $\mathrm{Sb} 3-\mathrm{S} 6-\mathrm{Sb} 4$ & $92.68(4)$ \\
\hline $\mathrm{N} 1-\mathrm{Mn}-\mathrm{N} 6$ & $104.66(15)$ & $\mathrm{Sb} 4-\mathrm{S} 7-\mathrm{Sb} 3$ & $94.02(4)$ \\
\hline $\mathrm{N} 3-\mathrm{Mn}-\mathrm{N} 6$ & $90.12(15)$ & & \\
\hline
\end{tabular}

Symmetry codes: (i) $-x, y-\frac{1}{2}, \frac{3}{2}-z$; (ii) $1+x, y, z$; (iii) $1-x, y-\frac{1}{2}, \frac{3}{2}-z$; (iv) $1-x, \frac{1}{2}+y, \frac{3}{2}-z ;(\mathrm{v})-x, \frac{1}{2}+y, \frac{3}{2}-z ;$ (vi) $x-1, y, z$.

The $\mathrm{H}$ atoms were positioned with idealized geometry [C$\mathrm{H}($ methylene $)=0.97 \AA$ and $\mathrm{N}-\mathrm{H}($ amine $)=0.90 \AA]$ and refined with fixed isotropic displacement parameters $\left[U_{\text {iso }}(\mathrm{H})=1.2 U_{\text {eq }} \mathrm{C}_{\text {methylene }}\right.$ and $\mathrm{C}($ amine $)=1.2 U_{\mathrm{eq}} \mathrm{N}$ (amine $\left.)\right]$ using a riding model.

Data collection: IPDS (Stoe \& Cie, 1998); cell refinement: IPDS; data reduction: IPDS; $\operatorname{program}(\mathrm{s})$ used to solve structure: SHELXS97 (Sheldrick, 1997); program(s) used to refine structure: SHELXL97 (Sheldrick, 1997); molecular graphics: DIAMOND (Brandenburg, 1999); software used to prepare material for publication: CIFTAB (Sheldrick, 1997).

Financial support by the state of Schleswig-Holstein and the Deutsche Forschungsgemeinschaft (DFG) is gratefully acknowledged. 


\section{References}

Bensch, W. \& Schur, M. (1997). Z. Naturforsch. Teil B, 52, 405-409. Brandenburg, K. (1999). DIAMOND. Release 2.1e. Crystal Impact GbR, Bonn, Germany.

Cordier, G., Schäfer, H. \& Schwidetzky, C. (1984). Z. Naturforsch. Teil B, 39, 131-134.

Dittmar, G. \& Schäfer, H. (1977). Z. Anorg. Allg. Chem. 437, 183-187.

Dittmar, G. \& Schäfer, H. (1978a). Z. Anorg. Allg. Chem. 441, 93-97.

Dittmar, G. \& Schäfer, H. (1978b). Z. Anorg. Allg. Chem. 441, 98-102.

Eisenmann, B. \& Schäfer, H. (1979). Z. Naturforsch. Teil B, 34, 383-385.

Ellermeier, J. \& Bensch, W. (2002). Monatsh. Chem. 133, 945-957.

Graf, H. A. \& Schäfer, H. (1972). Z. Naturforsch. Teil B, 27, 735-739.

Pfitzner, A. \& Kurowski, D. (2000). Z. Kristallogr. 215, 373-376.

Schaefer, M., Näther, C. \& Bensch, W. (2003). Solid State Sci. 5, 1135-1139.

Schur, M. \& Bensch, W. (1997). Eur. J. Solid State Inorg. Chem. 34, 457-466.

Sheldrick, G. M. (1997). SHELXS97, SHELXL97 and CIFTAB. University of Göttingen, Germany.

Sheldrick, W. S. \& Häusler, H.-J. (1988). Z. Anorg. Allg. Chem. 557, 105-111.

Stähler, R., Näther, C. \& Bensch, W. (2003). J. Solid State Chem. 174, 264-275.

Stephan, H.-O. \& Kanatzidis, M. G. (1997). Inorg. Chem. 36, 6050-6057.

Stoe \& Cie (1998). X-SHAPE (Version 1.03), X-RED (Version 1.11) and IPDS (Version 2.89). Stoe \& Cie, Darmstadt, Germany.

Zelewsky, A. von (1996). Stereochemistry of Coordination Compounds. Chichester: Wiley.

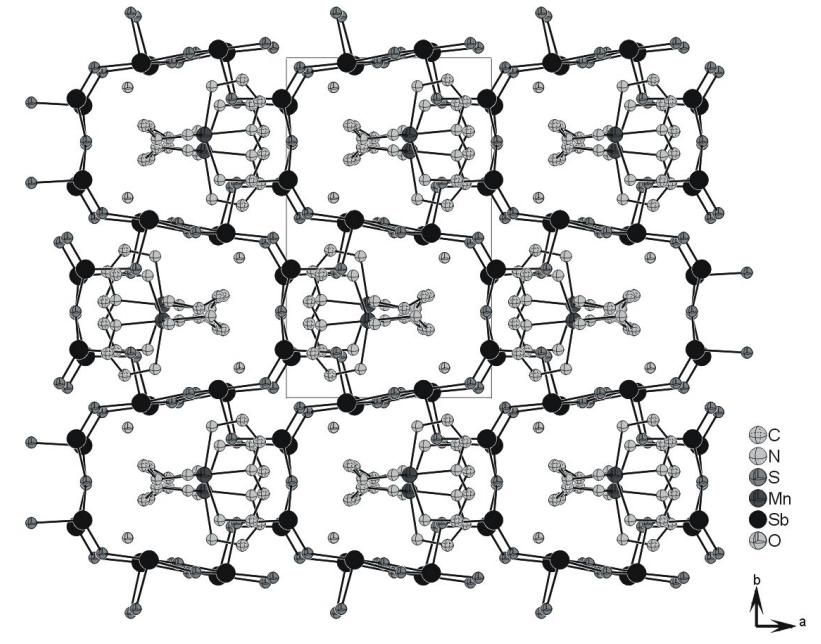

Figure 2

Arrangement of the cations and anions in $\left[\mathrm{Mn}(\operatorname{dien})_{2}\right] \mathrm{Sb}_{4} \mathrm{~S}_{7} \cdot 0.5 \mathrm{H}_{2} \mathrm{O}$, viewed along [001]. The cations are located above and below the $\mathrm{Sb}_{8} \mathrm{~S}_{8}$ rings. The $\mathrm{H}$ atoms of the dien ligands have been omitted for clarity. 